Now, by the original choice of $U, V_{0}$ has the property required by the theorem.

We remark that the same proof holds, with trivial modifications, for homology local connectivity.

It is an open question whether the neighborhood $V$ can be required to be connected.

YALE UNIVERSITY

\title{
UNCONDITIONAL CONVERGENCE IN BANACH SPACES
}

\section{S. KARLIN}

Introduction. This note investigates an apparent generalization of unconditionally convergent series $\sum x_{i}$ in weakly complete Banach spaces. A series of elements with $x_{i}$ in $E$ is said to be unconditionally convergent if for every variation of sign $\epsilon_{l}= \pm 1, \sum_{1}^{m} \epsilon_{l} x_{l}$ is convergent. This formulation of the definition of unconditional convergence is equivalent to that given by Orlicz [4]. ${ }^{1} \mathrm{We}$ call $\sum x_{i}$ unconditionally summable if there exists a finite row Toeplitz matrix $\left(b_{i k}\right)$ such that for every variation of sign $\sigma_{i}=\sum_{k=1}^{n_{i}} b_{i k} \sum_{l=1}^{k} \epsilon_{l} x_{l}$ converges. The fact that unconditional summability implies unconditional convergence is established in this note. Finally, applications to orthogonal functions are presented.

Preliminary lemmas. In what follows, $b_{i k}$ will denote an arbitrary finite row Toeplitz matrix.

LEMMA 1. If $S_{n}(\theta)=\sum_{1}^{m} a_{l} r_{l}(\theta)$ converges to an essentially bounded function $f(t)$, then $\left|\sum_{1}^{m} a_{n} r_{n}(\theta)\right| \leqq c$ almost everywhere. $\left(r_{n}(\theta)\right.$ denote the Rademacher functions.)

Proof. This is an immediate consequence of the result that

$$
\begin{aligned}
\left(\int_{0}^{1}\left(\max _{1 \leqq n \leqq m}\left|\sum_{1}^{n} a_{l} r_{l}(\theta)\right|\right)^{p} d \theta\right)^{1 / p} & \\
& \leqq C\left(\int_{0}^{1}\left|S_{m}(\theta)\right|^{p} d \theta\right)^{1 / p}, \quad 1 \leqq p \leqq \infty,
\end{aligned}
$$

Received by the editors April 30, 1947.

${ }^{1}$ Numbers in brackets refer to the references cited at the end of the paper. 
for all $m$ [3]. For, applying (1) for $p=\infty$, we get

$$
\left|\sum_{1}^{m} a_{l} r_{l}(\theta)\right| \leqq \max _{n}\left|\sum_{1}^{n} a_{l} r_{l}(\theta)\right| \leqq C\left|\sum_{1}^{\infty} a_{l} r_{l}(\theta)\right| \leqq c f(\theta) \leqq \gamma .
$$

LEMMA 2. If $\sigma_{i}=\sum_{k=1}^{m_{i}} b_{i k} \sum_{l=1}^{k} \epsilon_{l} a_{l}$ converges for every $\epsilon_{l}= \pm 1$, then $\sum_{1}^{\infty}\left|a_{l}\right|<\infty$.

Proof. We first establish that if $A_{i}=\sum_{k=1}^{m_{i}} A_{i k} \epsilon_{k}$ converges for every variation of sign $\epsilon_{k}= \pm 1$, then $\sum_{k=1}^{n_{i}}\left|A_{i k}\right| \leqq C$ for every $i$. If we observe that $\left\{\left(A_{i k}\right)=x_{i}\right\}_{i}$ is a sequence of elements in $(l)$ (space of absolutely convergent series), then the hypothesis implies that $x_{i}$ converges weakly for all functionals $f$ of the form $f=\left\{\epsilon_{k}\right\}$. A result of Banach [1] implies $x_{i}$ are strongly convergent and hence $\left\|x_{i}\right\|=\sum_{k=1}^{n_{i}}\left|A_{i k}\right| \leqq C$. We now complete the proof. Since

$$
\sigma_{i}=\sum_{k=1}^{n_{i}} b_{i k} \sum_{l=1}^{k} \epsilon_{l} a_{l}=\sum_{l=1}^{n_{i}} \epsilon_{l}\left[a_{l} \sum_{k=l}^{n_{i}} b_{i k}\right]=\sum_{l=1}^{n_{i}} \epsilon_{l} A_{i l}
$$

converges, we have, in view of the preceding, that

$$
\left|\sum_{k=1}^{n_{i}} b_{i k} \sum_{l=1}^{k} \epsilon_{l} a_{l}\right| \leqq \sum_{l=1}^{n_{i}}\left|a_{l} \sum_{k=l}^{n_{i}} b_{i k}\right|=\sum_{l=1}^{n_{i}}\left|A_{i l}\right| \leqq C .
$$

If we replace the $\epsilon_{l}$ by $r_{l}(\theta)$, then the hypothesis states that $\sigma_{i}(\theta)$ converges for every $\theta$. In virtue of a known result [6], this implies that $S_{m}(\theta)=\sum_{1}^{m} a_{l} r_{l}(\theta)$ converges almost everywhere. This fact combined with (2) yields easily by Lemma 1 that almost everywhere

$$
\left|\sum_{1}^{m} a_{n} r_{n}(\theta)\right| \leqq C, \quad n=1,2, \cdots \text {. }
$$

Using the independence of the Rademacher functions, we obtain for $\left|\lambda_{k}\right| \leqq 1$ that

$$
\begin{aligned}
\sum_{n=1}^{m} a_{n} \lambda_{n} & =\int_{0}^{1}\left(\sum_{1}^{m} a_{n} r_{n}(\theta)\right) \prod_{1}^{m}\left(1+\lambda_{k} r_{k}(\theta)\right) d \theta \\
& \leqq C \int_{0}^{1} \prod_{1}^{n}\left(1+\lambda_{k} r_{k}(\theta)\right) d \theta \leqq C .
\end{aligned}
$$

Choosing $\lambda_{n}=\operatorname{sign} a_{n}$, we get $\sum\left|a_{n}\right|<\infty$, which completes the proof. The hypothesis requiring every set $\left\{\epsilon_{l}\right\}$ in the lemma is necessary. For there exist numbers $a_{l}$ with $\sum a_{l}^{2}<\infty, \quad \sum\left|a_{l}\right|=\infty$, and $\sum a_{l} r_{l}(\theta)$ convergent almost everywhere, for example $a_{l}=1 / l$.

Another result needed in the same direction is the following: 
LEMma 3. If for almost all variation of sign

$$
\left|\sum_{k=1}^{n_{i}} b_{i k} \sum_{l=1}^{k} \epsilon_{l} a_{l}\right| \leqq C
$$

then $\sum\left|a_{l}\right|<\infty$.

PROoF. In view of the hypothesis and the proof of the preceding lemma, it will be sufficient to show that $\sigma_{i}=\sum_{k=1}^{n_{i}} b_{i k} \sum_{l=1}^{k} \epsilon_{l} a_{l}$ converges almost everywhere. We proceed to show this. The hypothesis implies that for $\sigma_{i}(\theta)=\sum_{k=1}^{n_{i}} b_{i k} \sum_{l=1}^{k} r_{l}(\theta) a_{l}$, we have for any $p>1$

$$
\int_{0}^{1}\left|\sigma_{i}(\theta)\right|^{p} d \theta \leqq \gamma
$$

From a result of Banach and Saks [5], we infer

$$
\lim _{n, m \rightarrow \infty} \int_{0}^{1}\left|\frac{1}{m} \sum_{k=1}^{m} \sigma_{i_{k}}(\theta)-\frac{1}{n} \sum_{k=1}^{n} \sigma_{i_{k}}(\theta)\right|^{p} d \theta=0 .
$$

This can be represented as a new Toeplitz matrix $E$ operating on $\sum_{1}^{m} a_{l} r_{l}(\theta)$ with $E\left(S_{m}(\theta)\right)=\left\{\sigma_{n}^{\prime}\right\}=\left\{(1 / n) \sum_{k=1}^{n} \sigma_{i_{k}}\right\}$. Consequently, (3) states that

$$
\int_{0}^{1}\left|\sigma_{n}^{\prime}(\theta)-\sigma_{m}^{\prime}(\theta)\right|^{p} d \theta \rightarrow 0 .
$$

But this yields the existence of a subsequence $\sigma_{n_{k}}^{\prime}(\theta)$ which converges almost everywhere. As above, this can be represented as $\left\{\sigma_{n_{k}}^{\prime}(\theta)\right\}=E^{\prime}\left\{\sigma_{n}^{\prime}(\theta)\right\}=E^{\prime} E\left\{S_{n}(\theta)\right\}$ where $E^{\prime} E$ is a new Toeplitz matrix. Hence, we have exhibited a Toeplitz matrix which sums $S_{m}(\theta)$. As in Lemma 1 this implies that $S_{m}(\theta)$ converges almost everywhere. Q.E.D.

The theorems. We now establish several results on unconditional convergent series in a Banach space $E$.

We assume that $E$ is weakly complete.

Theorem 1. If for $x_{i} \in E, \sum x_{i}$ is summable weakly by a Toeplitz matrix for every variation of sign, that is,

$$
\sigma_{i}=\sum_{k=1}^{n_{i}} b_{i k} \sum_{l=1}^{k} \epsilon_{l} x_{l}
$$

converges weakly as elements, then $\sum x_{l}$ is unconditionally convergent. 
Proof. In view of the fact that $E$ is weakly complete, it is sufficient to demonstrate that $\sum\left|f\left(x_{l}\right)\right|<\infty$ for every functional $f$ [see [4]). If $f$ denotes an arbitrary functional and $a_{i}=f\left(x_{i}\right)$, the hypothesis implies that, for every variation of $\operatorname{sign} \epsilon_{l}= \pm 1, \sum_{k=1}^{n_{i}} b_{i_{k}} \sum_{l=1}^{k} \epsilon_{l} a_{l}$ converges. If we apply Lemma 2, we obtain that

$$
\sum\left|a_{l}\right|<\infty \text { or } \sum\left|f\left(x_{l}\right)\right|<\infty .
$$

The following similar result can be established with the use of Lemma 3.

THEOREM 2. If $E$ is weakly complete and $\left\|\sum_{k=1}^{n_{i}} b_{i k} \sum_{l=1}^{k} \epsilon_{l} x_{l}\right\| \leqq C$ for almost all variation of signs, then $\sum x_{l}$ is unconditionally convergent.

A final result in this connection is the following. We assume that $E$ is weakly complete.

TheOREM 3. If for every rearrangement there exists a positive Toeplitz matrix with $\sum_{k=1}^{n_{i}} b_{i k} \sum_{l=1}^{k} x_{q(l)}$ convergent for $x_{l}$ in $E$, then $\sum x_{l}$ converges unconditionally.

Proof. If $f\left(x_{l}\right)=a_{l}$, we assert that $\sum\left|a_{l}\right|<\infty$. Otherwise there exists a rearrangement with $\sum a_{n(q)}=\infty$ and $\sum_{1}^{m} a_{n(q)}>0$ for every $m$. This implies that, for any positive Toeplitz matrix, $\sum_{k=1}^{n_{i}} b_{i k}$ $\sum_{1}^{k} a_{n(q)} \rightarrow \infty$ which contradicts the hypothesis. The remainder of the proof is straightforward.

Applications. We shall now apply these results to complete orthogonal systems of functions. It is customary in treating bounded orthogonal systems to assume that the Lebesque kernel is summable by a finite row Toeplitz matrix, that is

$$
\int_{0}^{1}\left|\sum_{k=1}^{n_{i}} b_{i k} \sum_{l=1}^{k} \phi_{l}(t) \phi_{l}(\theta)\right| d t \leqq C
$$

[2] almost everywhere in $\theta$. We make this assumption and establish the following theorem.

THEOREM 4. If for every variation of sign $\epsilon_{l}= \pm 1,\left\{\epsilon_{l} a_{l}\right\}$ are the Fourier coefficients with respect to $\left\{\phi_{k}(t)\right\}$ of a function in $L^{p}(p \geqq 1)$, then $\left\{d_{l} a_{l}\right\}$ are the Fourier coefficients of a function in $L^{p}$ for every sequence of numbers $d_{k}$ with $\left|d_{k}\right| \leqq 1$.

We first demonstrate a lemma.

LEMMA. If $\sum x_{i}$ converges unconditionally, then $\left\|\sum_{1}^{m} d_{l} x_{l}\right\| \leqq C$ for all $d_{l}$ with $\left|d_{l}\right| \leqq 1$. 
Proof. The hypothesis implies that

$$
\left\|\sum_{1}^{m} \epsilon_{l} x_{l}\right\| \leqq C
$$

for all $m$ and $\epsilon_{l}= \pm 1$. For each $m$ and $l=1, \cdots, m$ with $\left|d_{l}\right| \leqq 1$ there exists a functional $f_{m}$ with $\left|f_{m}\right|=1$ such that

$$
\left\|\sum_{l=1}^{m} d_{l} x_{l}\right\|=f_{m}\left(\sum_{1}^{m} d_{l} x_{l}\right) \text {. }
$$

We obtain now using (5) and (6)

$$
\begin{aligned}
\left\|\sum_{1}^{m} d_{l} x_{l}\right\| & =\left|\sum_{1}^{m} d_{l} f_{m}\left(x_{l}\right)\right| \leqq \sum_{1}^{m}\left|f_{m}\left(x_{l}\right)\right| \\
& =\sum_{1}^{m} \epsilon_{l} f_{m}\left(x_{l}\right)=f_{m}\left(\sum_{1}^{m} \epsilon_{l} x_{l}\right) \\
& \leqq\left|f_{m}\right|\left\|\sum_{1}^{m} \epsilon_{l} x_{l}\right\| \leqq C .
\end{aligned}
$$

Proof of Theorem 4. Let $\sigma_{i}(t)=\sum_{k=1}^{n_{i}} b_{i k} \quad \sum_{l=1}^{k} \epsilon_{l} a_{l} \phi_{l}(t)$, then in virtue of the criterion that a sequence of real numbers be the Fourier coefficients of a function in $L^{p}[2]$, we conclude that $\sum a_{l} \phi_{l}(t)$ is unconditionally summable in $L^{p}$. Applying Theorem 1 , we obtain that $\sum a_{l} \phi_{l}(t)$ is unconditionally convergent. The above lemma implies that $\sum_{1}^{m} d_{l} a_{l} \phi_{l}(t)$ is uniformly bounded in $L^{p}$ for any fixed set of $d_{l}$. Applying the criterion for determining Fourier coefficient in $L^{p}$ gives us the result.

\section{REFERENCES}

1. S. Banach, Théorie des operations linéaires, Warsaw, 1932, pp. 138-139.

2. S. Kaczmarz and H. Steinhaus. Theorie der Orthogonalreihen, Warsaw, 1935, chap. 5.

3. S. Marcienkiewicz and A. Zygmund, Quelques théorèmes sur les fonctions independantes, Studia Mathematica vol. 7 (1938) p. 113.

4. W. Orlicz, Beitrage zur Theorie der Orthogonolentruckbringen. II, Studia Mathematica, vol. 1 (1929) pp. 241-255.

5. S. Saks and S. Banach, Sur la convergence forte dons les champs $L^{p}$, Studia Mathematica vol. 2 (1930) p. 51.

6. A. Zygmund, Trigonometrical series, 1935, chap. 5, p. 119-127.

Princeton University 